\title{
Multi-source Features and Support Vector Machine for Heart Rhythm Classification
}

\author{
Chengyu Liu ${ }^{1,2}$, Qiao Li ${ }^{2}$, Pradyumna B. Suresh ${ }^{3}$, Adriana Vest ${ }^{2}$, Gari D. Clifford ${ }^{2,4}$ \\ ${ }^{1}$ School of Instrument Science and Engineering, Southeast University, Nanjing, China \\ ${ }^{2}$ Department of Biomedical Informatics, Emory University, Atlanta, USA \\ ${ }^{3}$ School of Electrical and Computer Engineering, Georgia Institute of Technology, Atlanta, USA \\ ${ }^{4}$ Department of Biomedical Engineering, Georgia Institute of Technology, Atlanta, USA
}

\begin{abstract}
The PhysioNet/CinC Challenge 2017 provides over 8,528 short single channel ECG recordings for the classification of rhythms as normal sinus rhythm, AF, other rhythm, or too noisy. We present a support vector machine (SVM)-based heart rhythm classifier that leverages features based on rhythm, morphology and arrhythmia characteristics of the ECG. Four benchmark signal quality features, eleven rhythm features, fifteen morphology features, six arrhythmia features and four novel abnormality indices, were presented to the libSVM software package with 10 fold cross-validation. The SVM parameters were tuned using the modified cuckoo search (MCS) algorithm during the training.

Since two of the authors were involved in the challenge, this is an unofficial entry. Without the MCS parameter optimization, the results for the mean $F_{I}$ measures from the 10 fold cross validation on the training set were $0.911,0.826,0.728$ and 0.852 for normal sinus rhythm, AF rhythm, other rhythm and noise respectively, resulting in a $F_{1}$ score of 0.822 . With MCS parameter optimization, the mean $F_{1}$ measures on the training set increased to $0.921,0.835,0.739$ and 0.870 respectively, resulting in a $F_{1}$ score of 0.832 . The final $F_{1}$ measures on the test set were $0.914,0.805,0.691$ and 0.737 for normal sinus rhythm, AF rhythm, other rhythm and noise respectively, resulting in a final $F_{1}$ score of 0.803 .
\end{abstract}

\section{Introduction}

Atrial fibrillation (AF) is the most common sustained cardiac arrhythmia, occurring in $1-2 \%$ of the general population and is associated with significant mortality and morbidity $[1 ; 2]$. Accurate detection for AF, especially from a variety of different arrhythmia types, is still challenging since some other arrhythmia types have the similar rhythm with AF. The PhysioNet/CinC Challenge
2017 provides a chance for the classification of rhythms as normal sinus rhythm, AF, other rhythm, or too noisy, from a short single channel ECG recordings. The details about the challenge can refer to [3].

In this study, we proposed a support vector machine (SVM)-based heart rhythm classifier that leverages features based on timing, morphology and spectral characteristics of the ECG, aiming to see how well when performing the common multi-source features and SVM for $\mathrm{AF}$ classification. We tried to use as many types of different $\mathrm{AF}$ features as possible since the previous studies confirmed that the multi-source features based machine learning approaches can provide an enhanced performance on AF detection [4-6].

\section{Methods}

\subsection{Data}

Data to support development and evaluation of challenge entries were collected by AliveCor device and provided freely via PhysioNet/CinC Challenge 2017 [3]. The data set includes 12,186 single lead ECG recordings lasting from $9 \mathrm{~s}$ to just over $60 \mathrm{~s}$, with a sample rate of $300 \mathrm{~Hz}$. The opened training set contains 8,528 recordings and the hidden test set (3,658 recordings) is unavailable to the public. Each recording was labeled as one of four types: normal sinus rhythm, $\mathrm{AF}$, an alternative rhythm, or is too noisy to be classified. The labels were initially annotated by AliveCor device and then a few of them were manually corrected by the challenge organizers and experts. Detailed data profile can refer to [3].

\subsection{Method description}

Figure 1 shows the block diagram of the proposed SVM and multiple feature-based method, which consisted of four steps:

- Step 1: signal quality assessment for each $10 \mathrm{~s}$ 
ECG episode.

- Step 2: feature calculation for the ECG episodes with good signal quality. Detailed feature definitions are given in section 2.3.

- Step 3: SVM (libsvm) classification using default parameter setting.

- Step 4: SVM (libsvm) classification using optimized parameter setting by using the modified cuckoo search algorithm [7;8].

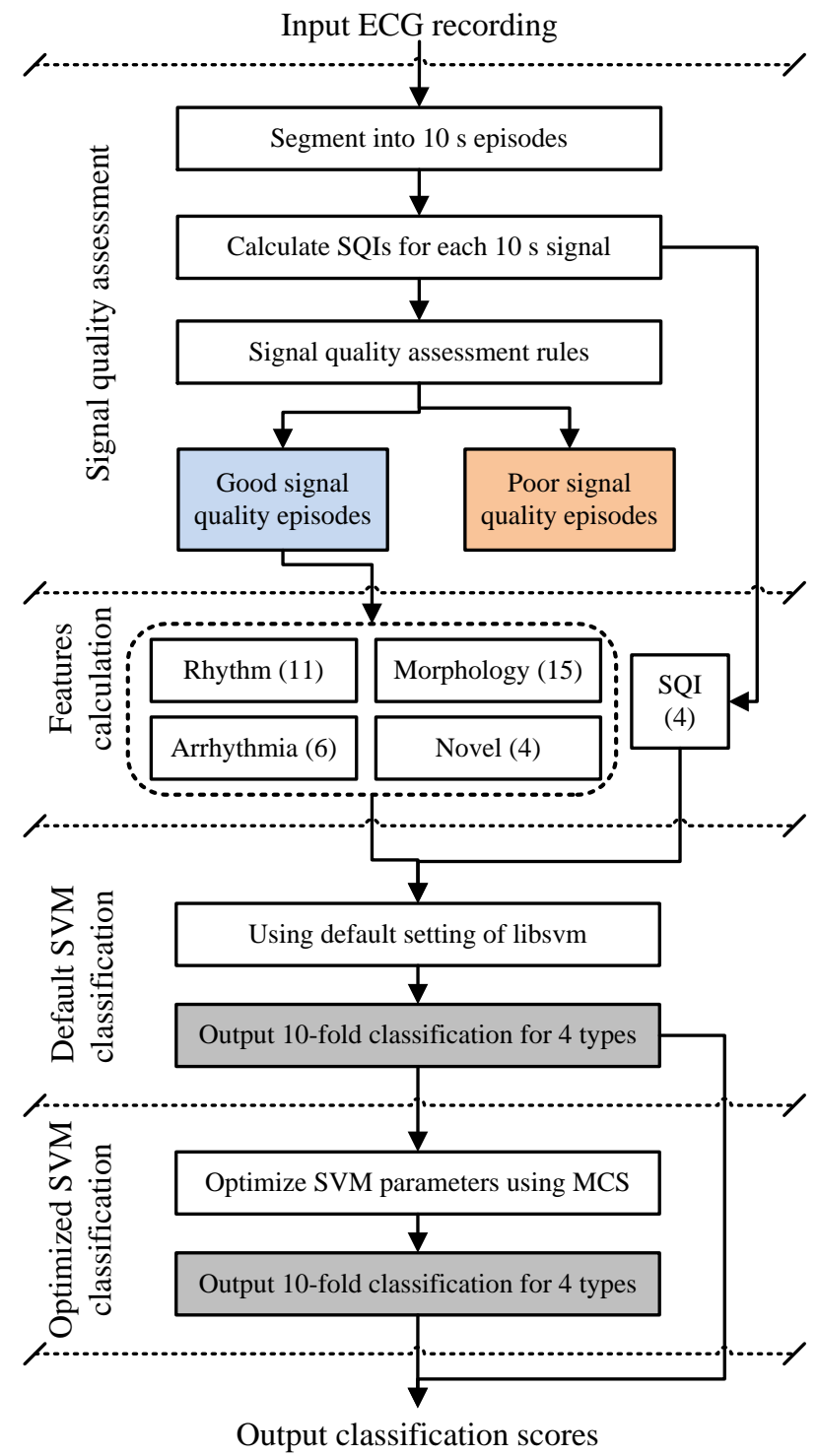

Figure 1. Block diagram of the proposed SVM and multiple feature-based method.

\subsection{Features}

We considered the features from five information sources. Signal quality index (SQI) was used as the first information source. The information about the heart rhythm, waveform morphology for QRS complex, $\mathrm{P}$ wave and $\mathrm{T}$ wave, if arrhythmia is presented, and the new developed novel features were used as the other four information sources.

A. Signal quality features (4) $[9 ; 10]$

- bSQI: percentage of beats detected by 'jqrs' that were also detected by Pan\&Tompkins method.

- sSQI: third moment (skewness) of the waveform distribution.

- kSQI: fourth moment (kurtosis) of the waveform distribution.

- fSQI: ratio of power $\mathrm{P}(5-14 \mathrm{~Hz}) / \mathrm{P}(5-40 \mathrm{~Hz})$ from spectrum analysis of burg method.

B. Rhythm features (11)

- MAD: thresholding on the median absolute deviation of RR intervals [11].

- AFEv: AF feature based on the analysis of Lorenz plot analysis [12].

- NFEn: normalized fuzzy entropy of the RR interval time series [13].

- noRR: number of RR intervals.

- mRR: mean value of RR intervals.

- minRR: minimum value of RR intervals.

- maxRR: maximum value of RR intervals.

- medHR: median value of heart rate calculate from each RR interval.

- SDNN: standard deviation of RR intervals.

- PNN50: mean value of RR intervals.

- RMSSD: root-mean square differences of RR intervals.

C. Morphology features for QRS complexes (5)

- mAmp: mean value of amplitudes of QRS complexes.

- sdAmp: standard deviation of amplitudes of QRS complexes.

- rAmp: ratio of $85 \%$ and $15 \%$ fractiles of the sorted amplitudes of QRS complexes.

- scovMor: correlation coefficient of QRS complexes in a small window $(200 \mathrm{~ms})$.

- lcovMor: correlation coefficient of QRS complexes in a large window (400 ms).

D. Morphology features for $\mathrm{P}$ and $\mathrm{T}$ waves (10)

- mAmpP \& sdAmpP: mean and SD of P wave amplitudes

- mP2QRS: mean of P amplitude/QRS amplitude

- sdP2QRS: SD of P amplitude/QRS amplitude

- mAmpT \& sdAmpT: mean and SD of T wave amplitudes

- mT2QRS: mean of T amplitude/QRS amplitude

- sdT2QRS: SD of T amplitude/QRS amplitude

- QT \& QTc: QT and calibrated QT interval

E. Arrhythmia features (6)

- PAC1-3: three PAC prediction indices based on impulse rejection filters

- PVC1-3: three PVC prediction indices based on frequency domain filters

F. Novel features (4) 
- MAI: morphology abnormal index for single beat ECG waveforms.

- TAI: template abnormal index for QRS complexes.

- AAI: amplitude abnormal index for single beat ECG waveforms.

- IAI: interval abnormal index for RR interval time series.

\subsection{SVM classifier}

The matrix of the forty features for the 8,528 training set recordings was calculated. We used the open-source libsvm software package to train the feature matrix and learn the SVM models [14]. Ten-fold cross-validation method was used to enhance the generalization ability of the trained SVM models. The default parameter setting for training SVM models were used as: kernel function: radial basis function; gamma parameter $\gamma$ in kernel function: $1 / N_{\text {feature }}\left(N_{\text {feature }}=40\right.$ here $)$; cost parameter $C: 1$; weights for parameters $C$ in each of the four classification type: 1 .

Then, we kept using the 10 -fold cross validation and using the kernel function of SVM as radial basis function, and tuned other SVM parameters using the modified cuckoo search (MCS) algorithm [7;8]. The objective function was set to maximize the mean $F_{1}$ measure for the first three rhythm types (normal, AF and other rhythm).

\subsection{Scoring mechanism}

The scoring for this challenge uses a $F_{1}$ measure. For each of the four classification type, $F_{1}$ is defined as:

- Normal rhythm: $F_{1 n}=\frac{2 \times N n}{\sum N+\sum n}$

- AF rhythm: $F_{1 a}=\frac{2 \times A a}{\sum A+\sum a}$

- Other rhythm: $F_{1 o}=\frac{2 \times O o}{\sum O+\sum o}$
- Noisy: $F_{1 p}=\frac{2 \times P p}{\sum P+\sum p}$

The final challenge score was generated as [3]:

$$
F_{1}=\frac{F_{1 n}+F_{1 a}+F_{1 o}+F_{1 p}}{4}
$$

\section{Results}

\subsection{SVM classification using default parameter setting}

The left half of the Table 1 shows the results from the 10 -fold cross validation for the training set when using the default parameter setting for training SVM model. Mean $F_{1}$ results are $0.911,0.826,0.728$ and 0.852 for normal sinus rhythm, AF rhythm, other rhythm and noisy types respectively, resulting in a mean $F_{1}$ of 0.822 . Normal type hold the largest $F_{1}$ measure of 0.911 , followed by the noisy class of 0.852 , and the AF type of 0.826 . Other type had the lowest largest $F_{1}$ value of 0.728 . The standard deviation (SD) values of the $F_{1}$ results for the four types are $0.019,0.045,0.026$ and 0.027 respectively. The total $\mathrm{SD}$ for the mean $F_{1}$ results from the 10-fold cross validation is 0.020 .

\subsection{SVM classification using MCS optimized parameter setting}

Results for the training set using the optimized SVM parameters were shown in the right half of the Table 1. Mean $F_{1}$ results from the 10 -fold cross validation are $0.921,0.835,0.739$ and 0.870 for normal sinus rhythm, AF rhythm, other rhythm and noisy types respectively, resulting in a mean $F_{1}$ of 0.832 . Compared with using the default SVM parameter setting, using the optimized SVM parameter enhanced the mean $F_{1}$ results for each of the four classification types, by $0.010,0.009,0.011$ and 0.018 respectively. The SD values of the $F_{1}$ results are 0.012 ,

Table 1. Results of the multi-source features and SVM-based algorithm on training (10-fold cross validation) and test sets.

\begin{tabular}{|c|c|c|c|c|c|c|c|c|c|c|c|}
\hline \multirow{2}{*}{ Set } & \multirow{2}{*}{ Fold } & \multicolumn{5}{|c|}{ SVM with default parameters } & \multicolumn{5}{|c|}{ SVM with MCS optimized parameters } \\
\hline & & Normal & $\mathrm{AF}$ & Other & Noisy & Total & Normal & $\mathrm{AF}$ & Other & Noisy & Total \\
\hline \multirow{12}{*}{ Training } & 1 & 0.878 & 0.746 & 0.690 & 0.845 & 0.771 & 0.905 & 0.746 & 0.705 & 0.879 & 0.785 \\
\hline & 2 & 0.910 & 0.830 & 0.723 & 0.808 & 0.820 & 0.914 & 0.836 & 0.726 & 0.835 & 0.825 \\
\hline & 3 & 0.933 & 0.783 & 0.738 & 0.843 & 0.818 & 0.933 & 0.812 & 0.763 & 0.855 & 0.836 \\
\hline & 4 & 0.899 & 0.871 & 0.736 & 0.854 & 0.835 & 0.907 & 0.860 & 0.733 & 0.864 & 0.833 \\
\hline & 5 & 0.929 & 0.844 & 0.708 & 0.861 & 0.827 & 0.922 & 0.834 & 0.730 & 0.871 & 0.829 \\
\hline & 6 & 0.925 & 0.777 & 0.753 & 0.856 & 0.818 & 0.920 & 0.773 & 0.750 & 0.859 & 0.814 \\
\hline & 7 & 0.905 & 0.875 & 0.762 & 0.864 & 0.847 & 0.940 & 0.895 & 0.768 & 0.877 & 0.867 \\
\hline & 8 & 0.886 & 0.841 & 0.748 & 0.908 & 0.825 & 0.922 & 0.866 & 0.767 & 0.922 & 0.852 \\
\hline & 9 & 0.923 & 0.878 & 0.687 & 0.861 & 0.829 & 0.940 & 0.898 & 0.714 & 0.890 & 0.851 \\
\hline & 10 & 0.924 & 0.818 & 0.741 & 0.817 & 0.828 & 0.915 & 0.829 & 0.733 & 0.845 & 0.826 \\
\hline & Mean & 0.911 & 0.826 & 0.728 & 0.852 & 0.822 & 0.921 & 0.835 & 0.739 & 0.870 & 0.832 \\
\hline & SD & 0.019 & 0.045 & 0.026 & 0.027 & 0.020 & 0.012 & 0.045 & 0.022 & 0.025 & 0.023 \\
\hline Test & & & & & & & 0.914 & 0.805 & 0.691 & 0.737 & 0.803 \\
\hline
\end{tabular}


$0.045,0.022$ and 0.025 respectively for the four types. The total SD for the mean $F_{1}$ results from the 10 -fold cross validation is 0.023 .

As shown in Table 1 , the final $F_{1}$ measures on the test set were $0.914,0.805,0.691$ and 0.737 for normal sinus rhythm, AF rhythm, other rhythm and noise respectively, resulting in a final $F_{1}$ score of 0.803 .

\section{Discussion and conclusion}

In this study, we present a support vector machine (SVM)-based heart rhythm classifier that leverages features based on rhythm, morphology and arrhythmia characteristics of the ECG. Features from benchmark signal quality, RR rhythm, QRS/P wave/T wave morphology, arrhythmia and novel abnormality indices were employed. The open-source libSVM software package with 10 fold cross-validation was used to test and validate the usefulness of the multiple sourced features. In addition, we tested the effect of tuning the SVM parameters by the modified cuckoo search (MCS) algorithm on the classification accuracy. Using the MCS parameter optimization, improved mean $F_{1}$ measure of 0.832 on the training set from the 10 fold cross validation was achieved. As comparison, without MCS parameter optimization, the mean $F_{1}$ measure on the training set from the 10 fold cross validation was 0.822 . When tested on the test set, a final $F_{1}$ score of 0.803 was achieved. Please note that we are unofficial entry since two of the authors were involved in the challenge.

The current study used multiple sourced features for the four classification tasks. We expect that, more features, including specific design for other rhythm type, VT/VF detection, spectrum/cepstral analysis, etc, could further enhance the classification performance. In addition, no feature selection step was used in the current study. Thus, in the next phase, selecting more efficient features and excluding the redundancy and relevance between features, could also be helpful to enhance the classification performance.

\section{Acknowledgements}

Chengyu Liu would like to thank the funding from the International Postdoctoral Exchange Programme of the National Postdoctoral Management Committee of China.

\section{References}

[1] Lip G Y H, Fauchier L, Freedman S B, Van Gelder I, Natale A, Gianni C, Nattel S, Potpara T, Rienstra M, Tse H, Lane D A. Atrial fibrillation. Nature Reviews Disease Primers. 2016; 2: 16016.

[2] Camm A J, Kirchhof P, Lip G Y, Schotten U, Savelieva I, Ernst S, Van Gelder I C, Al-Attar N, Hindricks G, Prendergast B, Heidbuchel H, Alfieri O, Angelini A, Atar
D, Colonna P, De Caterina R, De Sutter J, Goette A, Gorenek B, Heldal M, Hohloser S H, Kolh P, Le Heuzey J Y, Ponikowski P, Rutten F H. Guidelines for the management of atrial fibrillation: the Task Force for the Management of Atrial Fibrillation of the European Society of Cardiology (ESC). Eur Heart J. 2010; 31: 2369-2429.

[3] Clifford G D, Liu C Y, Moody B, Lehman L, Silva I, Li Q, Johnson A E, Mark R G. AF Classification from a short single lead ECG recording: the PhysioNet/Computing in Cardiology Challenge 2017. Computing in Cardiology. 2017; 44: in press.

[4] Li Q, Liu C Y, Oster J, Clifford G D 2016 Machine Learning for Healthcare Technologies, ed D A Clifton (London: IET) pp 33-58

[5] Oster J, Clifford G D. Impact of the presence of noise on RR interval-based atrial fibrillation detection. J Electrocardiol. 2015; 48: 947-951.

[6] Colloca R, Johnson A E W, Mainardi L, Clifford G D. A support vector machine approach for reliable detection of atrial fibrillation events. Computing in Cardiology. 2013; 40: 1047-1050.

[7] Walton S, Hassan O, Morgan K, Brown M R. Modified cuckoo search: A new gradient free optimisation algorithm. Chaos, Solitons \& Fractals. 2011; 44: 710-718.

[8] Whitaker B W, Suresha1 P B, Liu C Y, Clifford G D, Anderson D V. Combining sparse coding and timedomain features for heart sound classification. Physiological Measurement. 2017; 38: 1701-1729.

[9] Clifford G D, Lopez D, Li Q, Rezek I. Signal quality indices and data fusion for determining acceptability of electrocardiograms collected in noisy ambulatory environments. Computing in Cardiology. 2011; 38: 285288.

[10] Li Q, Mark R G, Clifford G D. Robust heart rate estimation from multiple asynchronous noisy sources using signal quality indices and a Kalman filter. Physiol Meas. 2008; 29: 15-32.

[11] Linker D T. Long-term monitoring for detection of atrial fibrillation. US Patent. 2009; US20060084883:

[12] Sarkar S, Ritscher D, Mehra R. A detector for a chronic implantable atrial tachyarrhythmia monitor. IEEE Trans Biomed Eng. 2008; 55: 1219-1224.

[13] Liu C Y, Oster J, Reinertsen E, Li Q, Zhao L N, Nemati S, Clifford G D. A review of methods of entropy for atrial brillation detection. BioMedical Engineering OnLine. 2017; submitted.

[14] Chang C C, Lin C J. LIBSVM: A library for support vector machines. ACM Transactions on Intelligent Systems and Technology. 2011; 2: 1-27.

Address for correspondence.

Chengyu Liu

School of Instrument Science and Engineering

Southeast University

Nanjing, China

E-mail: bestlcy@sdu.edu.cn 